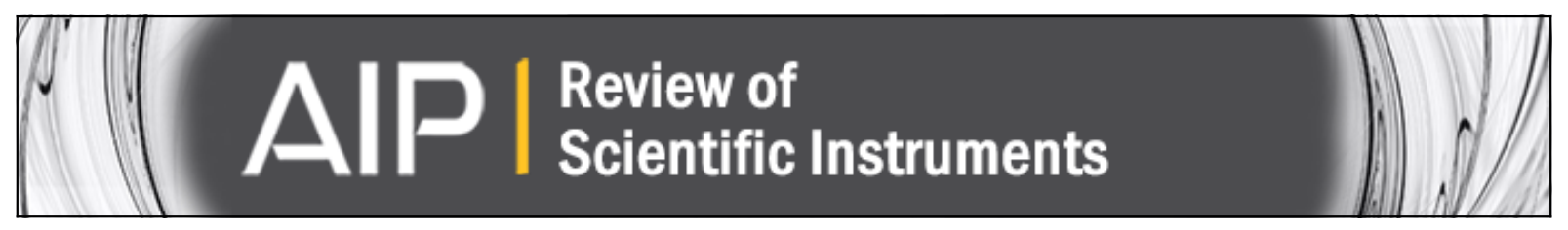

\title{
An atomic force microscope tip as a light source
}

Valentin Lulevich, Chris Honig, and William A. Ducker

Citation: Review of Scientific Instruments 76, 123704 (2005); doi: 10.1063/1.2149149

View online: http://dx.doi.org/10.1063/1.2149149

View Table of Contents: http://scitation.aip.org/content/aip/journal/rsi/76/12?ver=pdfcov

Published by the AIP Publishing

\section{AlP Re-register for Table of Content Alerts}




\title{
An atomic force microscope tip as a light source
}

\author{
Valentin Lulevich \\ Department of Chemistry, Virginia Tech, Virginia 24061 \\ Chris Honig \\ Department of Chemical and Biomolecular Engineering, University of Melbourne, VIC 3010 Australia \\ William A. Ducker ${ }^{\text {a) }}$ \\ Department of Chemistry, Virginia Tech, Virginia 24061 and \\ Department of Chemical and Biomolecular Engineering, University of Melbourne, VIC 3010 Australia
}

(Received 24 August 2005; accepted 14 November 2005; published online 21 December 2005)

\begin{abstract}
We present a simple method for causing the end of a silicon nitride atomic force microscope (AFM) tip to emit light, and we use this emitted light to perform scanning near-field optical microscopy. Illumination of a silicon nitride AFM tip by blue $(488 \mathrm{~nm})$ or green $(532 \mathrm{~nm})$ laser light causes the sharp part of the tip to emit orange light. Orange light is emitted when the tip is immersed in either air or water; and while under illumination, emission continues for a period of many hours without photobleaching. By careful alignment of the incident beam, we can arrange the scattered light to decay as a function of the tip-substrate separation with a decay length of 100-200 nm. The exponential decay of the intensity means that the emitted light is dominated by contributions from parts of the tip that are near the sample, and therefore the emitted orange light can be used to capture high-resolution near-field optical images in air or water. () 2005 American Institute of Physics.
\end{abstract}

[DOI: $10.1063 / 1.2149149$ ]

\section{INTRODUCTION}

An atomic force microscope (AFM) is a useful tool for high-resolution imaging ${ }^{1}$ and precise surface force measurements. ${ }^{2}$ A related technique, scanning near-field optical microscopy (SNOM), ${ }^{3}$ can be used to obtain highresolution optical images by collecting or emitting light from a sharp tip that is near the sample. The advantage of SNOM over AFM is that spectroscopic imaging of surfaces allows more facile identification of surface species than the force imaging that is performed in AFM. There are two basic types of SNOMs: those in which the tip acts as a waveguide and those in which the tip is used to scatter an evanescent wave that is produced at the surface of a sample (apertureless SNOM $)^{4,5}$

In this article, we present a simple method to make a regular gold-coated silicon nitride AFM tip emit light: when the tip is illuminated by a high intensity of light, it emits light of a different frequency. The frequency shift is very useful in SNOM applications because, by simple frequency filtering, one can obtain signals exclusively from an AFM tip. This makes the technique quite distinct from conventional apertureless SNOM, in which elastically scattered light is recorded. The contrast mechanism in apertureless SNOM is still a subject of debate, but it is thought that contrast arises from interference between scattering from the tip and the sample. ${ }^{6}$ By measuring the signal from the tip only, we aim to eliminate this interference. We show that high-

\footnotetext{
${ }^{\text {a) }}$ Author to whom all correspondence should be addressed; electronic mail: wducker@unimelb.edu.au
}

resolution optical images can be captured by analyzing the inelastically scattered light when a sample is scanned under an illuminated tip.

\section{EXPERIMENT}

The schematic of the experiment is shown in Fig. 1. An atomic force microscope (AFM, Asylum 3D, Santa Barbara) was mounted above an inverted optical microscope (Zeiss Axiovert, Carl Zeiss, Thornwood, NY). Laser light, reflected from a dichroic mirror, was focused on the AFM tip by an objective lens (N.A. 1.45, 60× TIRFM objective, Olympus, Japan). A high numerical aperture lens was used because it gives a high optical magnification of the tip, and provides the ability to use a high angle of refraction (relative to the normal). After the objective, the beam passes through a thinbottomed (0.1-0.15 mm) glass dish (MatTec, USA). A small drop of index matching liquid was placed between the lens and the glass dish. A thermoelectrically cooled diode laser (532 nm, $10 \mathrm{~mW}$, TECGL-10, World Star Tech., Canada) or an Ar-ion water-cooled laser (488 $\mathrm{nm}$ line of $1 \mathrm{~W}$, Innova Enterprise 2, model 610, CA, USA) was used as the light source. The beam on the cantilever or tip had an approximate diameter of 1-2 $\mu \mathrm{m}$. We used commercial silicon nitride AFM cantilevers (Veeco, USA) with integrated, hollow square-pyramidal $\mathrm{Si}_{3} \mathrm{~N}_{4}$ tips. The cantilever and tip were coated with $10 \mathrm{~nm} \mathrm{Cr}$ and $30 \mathrm{~nm} \mathrm{Au}$ on the side opposite the protruding tip.

Light scattered from the AFM tip was collected by the microscope objective and after passing though the dichroic mirror was collected by either (a) a photomultiplier tube (PMT) (Hitachi H5784-01) or (b) a fiber optical UV-vis spec- 


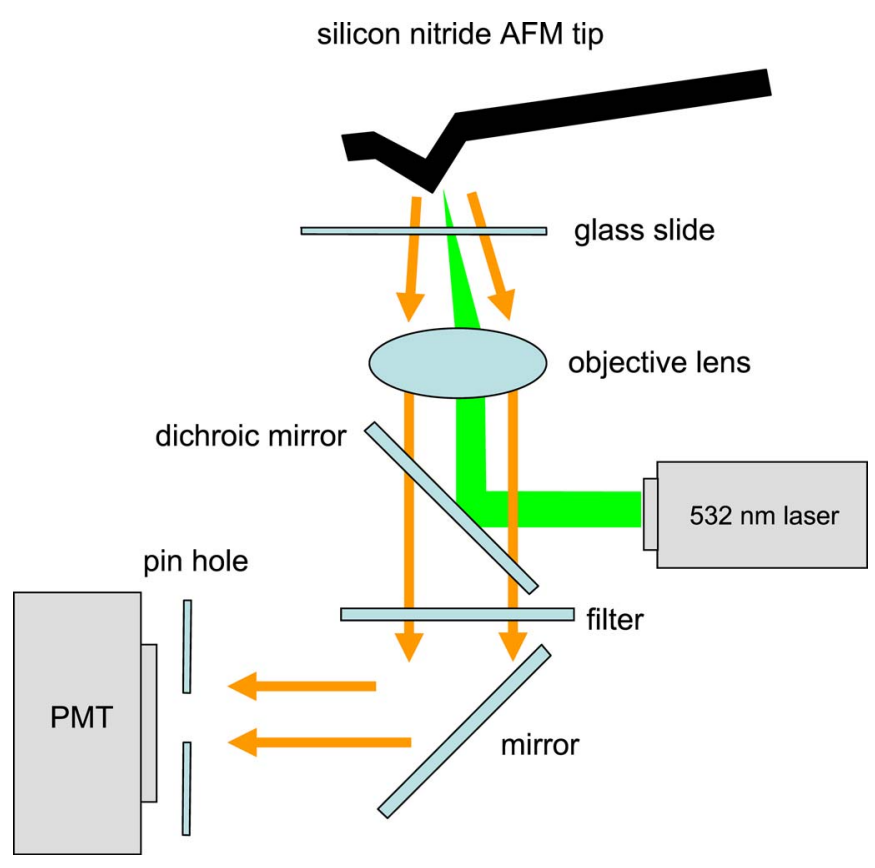

FIG. 1. (Color online) Schematic of the experiment. Parallel light from the laser is focused into a $1.0-0.5 \mu \mathrm{m}$ spot on the AFM tip by an objective lens. Inelastically scattered light is detected by a photomultiplier tube after passing through both the dichroic mirror and a filter passing $\lambda>540 \mathrm{~nm}$.

trometer (SD2000, Ocean Optics Inc., USA). In some experiments, the separation between the cantilever and the glass dish was varied $0.2-20 \mu \mathrm{m} / \mathrm{s}$ using a piezoelectric transducer and measured by a linear voltage-displacement transducer (LVDT). No speed dependence was observed. Unless otherwise specified, all experiments were performed in air. Spectra from Fig. 3 were obtained using a Horiba Jobin Yvon Triax 550 spectrometer.

\section{RESULTS AND DISCUSSION}

When we illuminate a commercial silicon nitride AFM tip in air with focused green $(532 \mathrm{~nm})$ light, the sharp end of the AFM tip (the part that is in close proximity to the sample during imaging) emits orange light. Figure 2 shows the emitted orange light when we have reduced the intensity of green light using both a dichroic mirror and a high-pass filter (cutoff frequency $540 \mathrm{~nm}$ ) that together reduce the $532 \mathrm{~nm}$ intensity by a factor of $10^{7}$. The frequency spectrum of the orange light shows a maximum at about $650 \mathrm{~nm}$. Most importantly for applications relating to microscopy, no photobleaching of the emission was observed, even over periods of hours.

Emission from the AFM tip is interesting because of applications to near-field microscopy, but we will first describe the far-field emission properties from the cantilever leg, which is about $600 \mathrm{~nm}$ thick and is coated with $10 \mathrm{~nm}$ $\mathrm{Cr}$ and $30 \mathrm{~nm} \mathrm{Au}$ on the side opposite the impinging laser beam. The cantilever leg also emits orange light when it is illuminated at $532 \mathrm{~nm}$, the spectrum of which is shown in Fig. 3. In the absence of the metallic coating, there is still emission, but the emission has a lower intensity and is more broad. Very similar emission was observed from silicon nitride particles (polydisperse, diameter 1-50 $\mu \mathrm{m}$ ). Recent

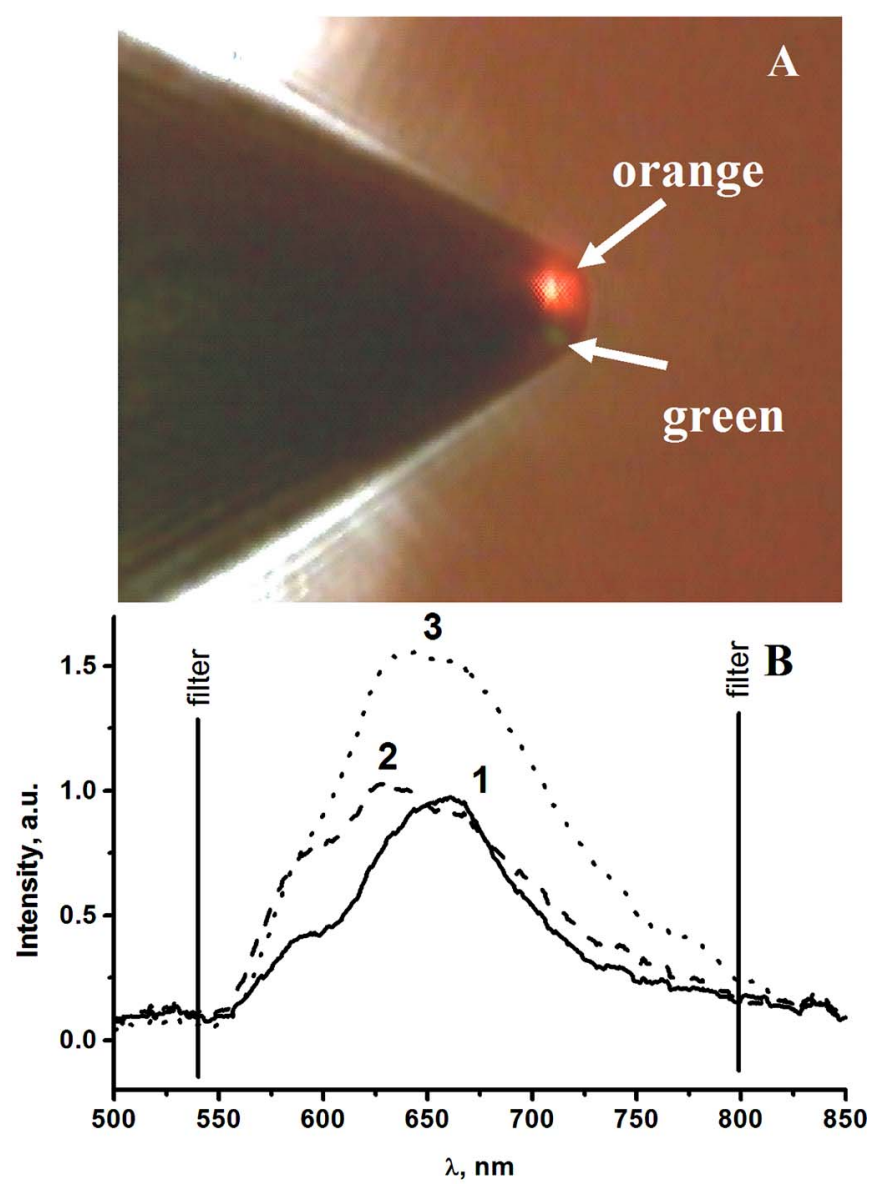

FIG. 2. (Color online) Inelastic scattering from AFM tip scattering is observed when the AFM tip is placed near a glass plate and is illuminated by the green (532 nm) laser. (a) Photograph of the end of the AFM cantilever in which the green light has been heavily filtered. (b) Spectrum of the emitted light under various conditions: 1 (solid), $532 \mathrm{~nm}$ excitation in air; 2 (dashed), $488 \mathrm{~nm}$ excitation in air; and 3 (dotted), $532 \mathrm{~nm}$ excitation in water.

related work has also shown that amorphous silicon nitride may contain nanocrystals of silicon, which are photoluminescent. ${ }^{7,8}$ Very thin $(20-35 \mathrm{~nm})$ single crystals of silicon nitride have also been shown to be photoluminescent. ${ }^{9}$ It has been suggested that the origin of the photoluminescence in silicon nitride may be dangling bonds or silicon nanoclusters within the silicon nitride. ${ }^{7-9}$

Gold is also photoluminescent but its role here appears to be enhancement of the silicon nitride emission: the goldcoated silicon nitride cantilever leg produces a sharper and more intense peak than either gold or silicon nitride.

The emission intensity is proportional to input intensity below about $2 \mathrm{~mW}$ of input power; at greater input power, the yield of orange light is less (Fig. 4). The linear dependence on incident intensity and then saturation is inconsistent with a nonlinear optical effect, despite the high power density of illumination by the laser beam (up to $10^{16} \mathrm{~W} / \mathrm{m}^{3}$ ). We are also able to eliminate blackbody emission as the source of the photoluminescence because the emission peak is too narrow and the wavelength of the peak is too short. (When the cantilever is immersed in liquid water, the peak corresponds to blackbody radiation at $4310 \mathrm{~K}$ whereas the tip temperature must be in the vicinity of $273-373 \mathrm{~K}$ ). Also, the 


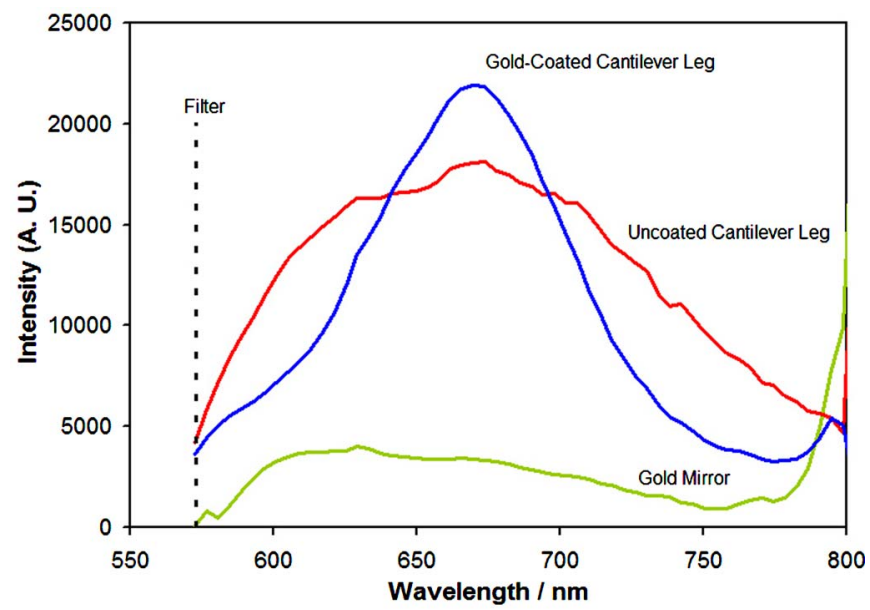

FIG. 3. (Color online) Emission spectra from AFM cantilever legs when illuminated with $532 \mathrm{~nm}$ light. The emission spectra for silicon nitride powder and a gold mirror are shown for comparison. The relative intensities of different samples in this figure are not comparable.

spectral peak does not change with laser power input, as one would expect for a blackbody heated with a laser. We are able to eliminate Raman scattering because there is no antiStokes emission. In the absence of other mechanisms, we conclude that the origin of the photoluminescence is probably fluorescence. Chemical impurities may be contributing to the fluorescence: we found some variation of the peak emission in the region $650-675 \mathrm{~nm}$ depending on the batch of cantilevers, but emission was always observed.

As stated earlier, photobleaching was not observed. In contrast, at the highest illumination power $(10 \mathrm{~mW})$, we occasionally produced visible damage to the edge of a goldcoated cantilever. By scanning the laser, we could produce damage lines on the cantilever. Such damaged regions produced a much greater intensity of photoluminescence (up to ten times the intensity from undamaged areas). This effect is useful for enhancing the signal in microscopy applications.

Returning now to emission from the AFM tip, Fig. 2 shows that orange emission is also observed under illumination from $488 \mathrm{~nm}$ light (Ar laser, $10 \mathrm{~mW}$ power), except that the emission frequency is shifted to a shorter wavelength; the maximum intensity is at about $625 \mathrm{~nm}$. The frequency shift between the illuminating light and the peak of the photoluminescence is not the same for the 488 and $532 \mathrm{~nm}$ sources, which confirms that the photoluminescence is not Raman scattering. Thus, by controlling the illumination frequency, we attain some control of the emission frequency. This will be useful for excitation of different chromophores.

We were also able to change the emission frequency by varying the thickness of gold. The standard silicon nitride cantilever is coated in $30 \mathrm{~nm}$ of gold on the side opposite the protruding tip. X-ray photoelectron spectroscopy (XPS) measurements show that the average thickness of gold on the inside of the AFM tip is much thinner $(\sim 5 \mathrm{~nm})$. After deposition of $40 \mathrm{~nm}$ of additional gold on the back side of the cantilever, the maximum in the spectrum shifts to the yellow range (about $600 \mathrm{~nm}$ ) and the scattered intensity is decreased by about 10 times.

AFM and SNOM find useful applications in aqueous so-
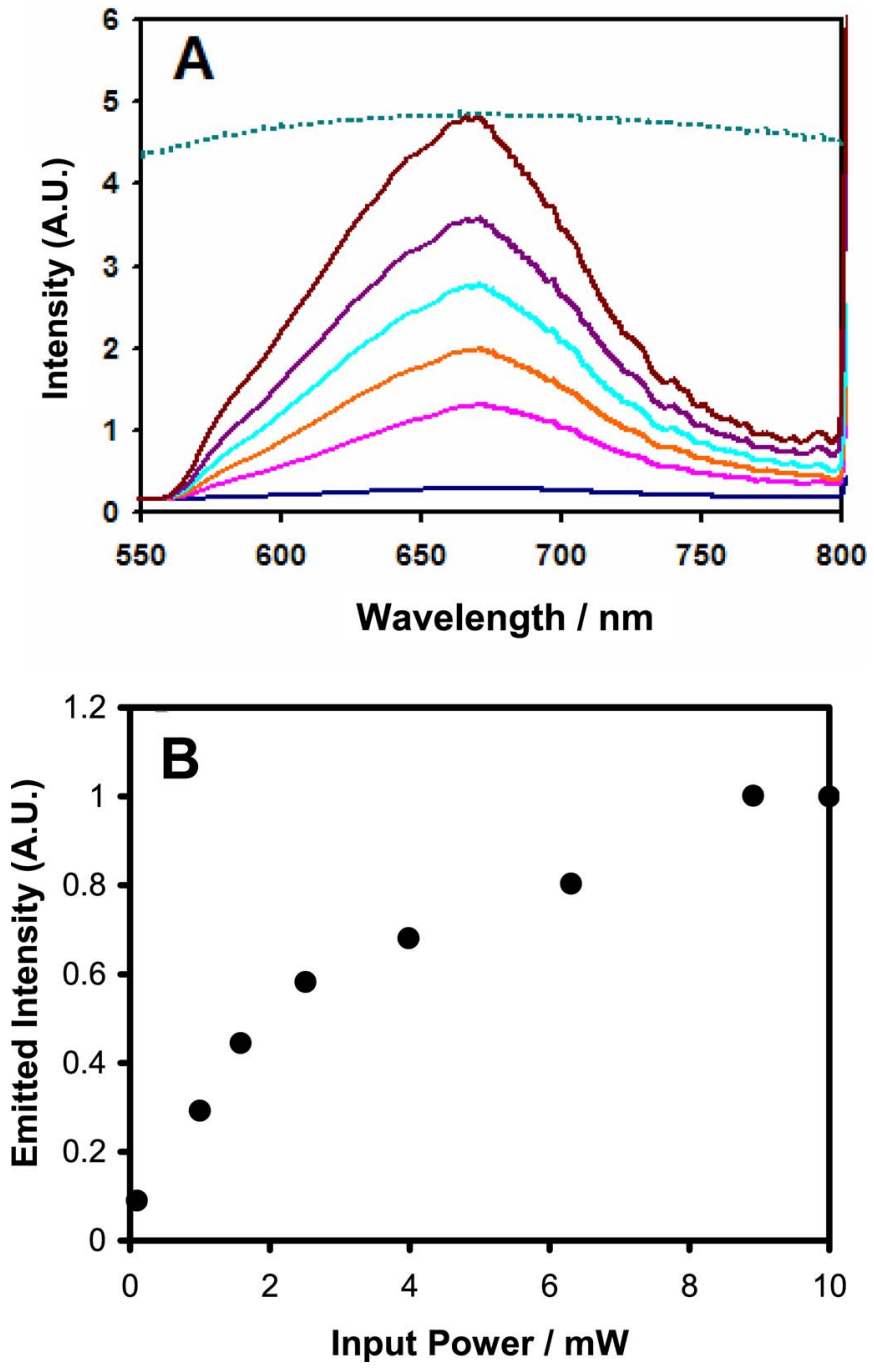

FIG. 4. (Color online) Light emitted from the silicon nitride cantilever leg in water as a function of input power at $532 \mathrm{~nm}$. (a) Spectrum. From top to bottom, the spectra are blackbody prediction at $4310 \mathrm{~K}$, measured spectrum at $6.3,4,2.5,1.6,1$, and $0.1 \mathrm{~mW}$ incident laser power. Note that the spectral shape does not change with input power. (b) Intensity of emitted light at $650 \mathrm{~nm}$.

lutions, so we examined the photoluminescence in water. At very high illumination intensities $\left(10^{16} \mathrm{~W} / \mathrm{m}^{3}\right)$, bubbles grow on both the tip and the glass sample plate, probably by optical cavitation. ${ }^{10}$ By illumination with a lower intensity of light, we are able to produce photoluminescence (see Figs. 2 and 4) while avoiding bubble formation and thereby produce emission of orange light from the tip under conditions that are useful to AFM and related techniques.

We have monitored the emission while the excitation laser is chopped at frequencies in the range 6-200 Hz. The emitted light is modulated at the same frequency. When the tip is not in contact with the glass plate, modulation of the incident irradiation results in modulation of the cantilever deflection with the same frequency. The force from the photon pressure $\left(\sim 3 \times 10^{-11} \mathrm{~N}\right)$ is too small to cause the deflection. This force is probably due to a thermal effect, either bending of the cantilever due to differential expansion of the gold coating relative to the silicon nitride, or expansion of the surrounding air. ${ }^{11}$ This modulation of forces on the tip 


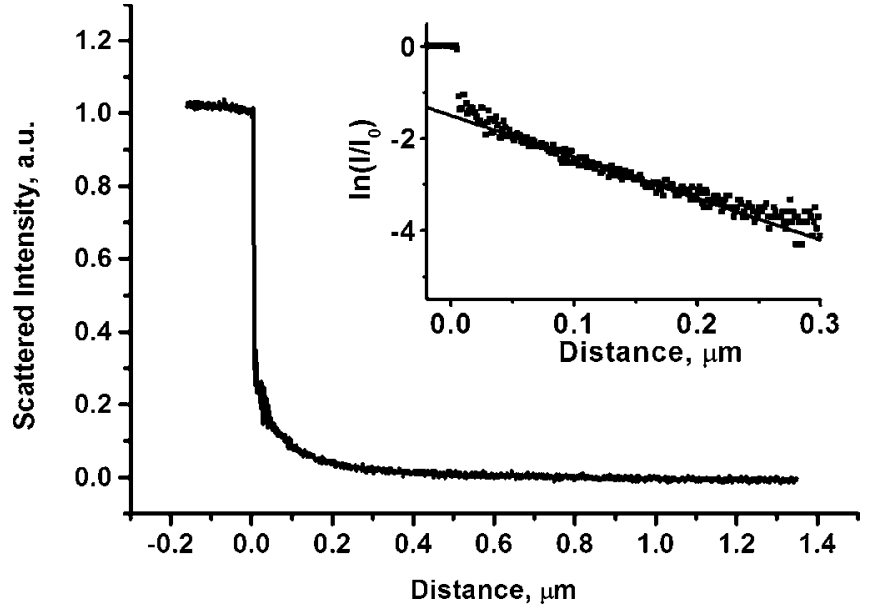

FIG. 5. Inelastic scattering as a function of the separation between an AFM tip and the glass plate. Data shown are for large $\left(80^{\circ}<\theta<90^{\circ}\right)$ refraction angles. The inset shows the same data on a log scale, as well as an exponential fit with a $110 \mathrm{~nm}$ decay length. The large increase in intensity at approximately zero distance in the lower figure is due to a mechanical instability that causes the tip to jump toward the plate. The intensity is no longer exponential because a surface force bends the spring, removing the correspondence between distance and separation. After the instability, the intensity no longer increases with decreasing distance because the tip is resting on the sample.

may be useful for AFM techniques that rely on cantilever vibration $^{12}$ (e.g., tapping mode AFM), for signal-to-noise reduction, or to control the effective stiffness of the cantilever. A static force is experienced for a constant illumination.

To be useful in near-field microscopy applications, the emission of light from the tip needs to be enhanced by proximity to a sample. If enhancement occurs near a sample, then optical output from the end of the AFM tip will dominate the emission, thereby localizing the emitted signal to a small area. This localization produces high lateral resolution in optical microscopy. We also measured the intensity of emitted orange light that is captured by our objective lens as a function of the separation between the tip and our sample (Fig. 5) under conditions where the angle of incidence for the illuminating light into the sample is clearly less than the critical angle: light that illuminates the tip is a transmitted wave rather than an evanescent wave. When the angle of refraction is less than about $80^{\circ}$ (relative to the normal), the dependence of the intensity on separation is rather weak, but when the angle is greater than $80^{\circ}$, the intensity profile is steep and exponential with a decay length of about 100-110 nm. This is a useful regime for near-field imaging of surfaces. Thus, the photoluminescence has the appropriate properties for high-resolution imaging. The angular dependence of the decay length is consistent with an evanescent wave produced during internal reflection within the AFM tip.

Figure 6 is a SNOM image of graphite particles on glass that was captured by illuminating the AFM tip with $532 \mathrm{~nm}$ light and measuring the intensity of 540-800 nm light while scanning the graphite sample under the tip. This method is seen to resolve small particles with a diameter of about $20 \mathrm{~nm}$.

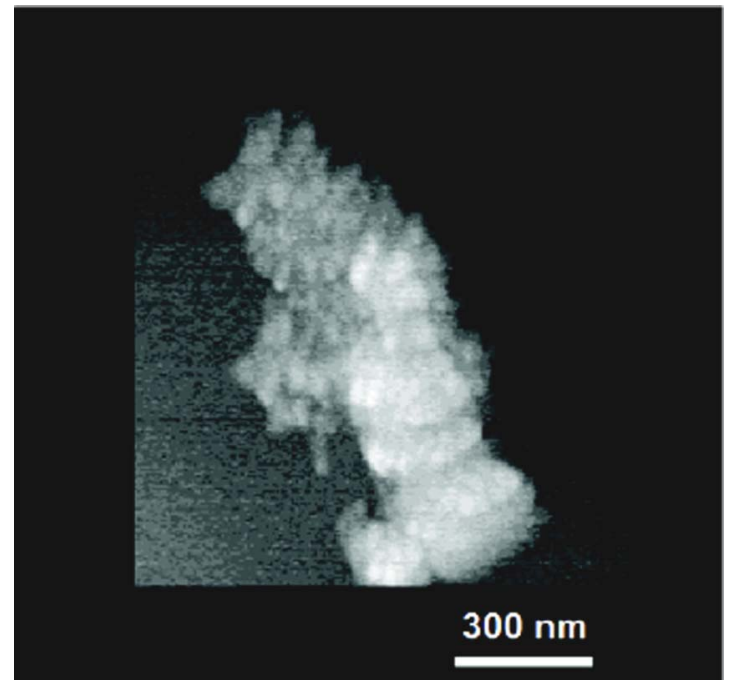

FIG. 6. (Color online) High-resolution SNOM image. This image was obtained by scanning a sample of (opaque) graphite particles under an AFM tip that is illuminated with $532 \mathrm{~nm}$ light and recording the intensity of light in the range $540-800 \mathrm{~nm}$. The image is given in negative contrast so that a low intensity of light collected by the PMT is shown as white: the opaque carbon particles block the transmission of the light.

\section{DISCUSSION}

In summary, it is easy to make a silicon nitride AFM tip emit light in air or water by simply illuminating the tip with green light $(532 \mathrm{~nm})$ that is transmitted through an AFM sample. The emitted light has an orange color $\left(\lambda_{\max } \sim 650 \mathrm{~nm}\right)$, so it can be separated from elastically scattered green light, and the intensity varies exponentially with the separation between the tip and the sample. This exponential decay is useful because it allows illumination of a small area under the AFM tip, and therefore it is useful for SNOM. In comparison to conventional apertureless SNOM (using elastic scattering), the inelastic-scattering method is easier to interpret because one does not need to worry about signals arising from the sample ${ }^{6}$ (provided that the sample is not photoluminescent). By simple addition of an illuminating source, a filter, and a light detector, one can convert a commercial AFM into an SNOM using mass-produced silicon nitride cantilevers. We anticipate that this device will be useful for imaging small particles and thin films in materials science and biological applications.

\section{ACKNOWLEDGMENTS}

The authors thank Spencer Clark, Randy Heflin, and Trevor Smith for experimental assistance, Clayton McKee for performing the XPS of the gold on the cantilever, Trevor Smith for the use of equipment in his laboratory, and Stephen Danforth for providing the silicon nitride powder. This work was funded by the Australian Research Council, Unilever (US), and the National Science Foundation Grant No. DMR0216129.

${ }^{1}$ G. Binnig, C. F. Quate, and C. Gerber, Phys. Rev. Lett. 56, 930 (1986).

${ }^{2}$ W. Ducker, T. J. Senden, and R. M. Pashley, Langmuir 8, 1831 (1992).

${ }^{3}$ H. Heinzelmann and D. W. Pohl, Appl. Phys. A 59, 89 (1994).

${ }^{4}$ N. F. van Hulst, N. P. Deboer, and B. Bolger, J. Microsc. 163, 117 (1991).

${ }^{5}$ N. F. van Hulst, M. H. P. Moers, O. F. J. Noordman, R. G. Tack, F. B. 
Segerink, and B. Boelger, Appl. Phys. Lett. 62, 461 (1993).

${ }^{6}$ J. Azoulay, A. Debarre, A. Richards, and P. Tchenio, Appl. Opt. 39, 129 (2000).

${ }^{7}$ V. A. Gritsenko, D. V. Gritsenko, Y. N. Novikov, R. W. M. Kwok, and I. Bello, J. Exp. Theor. Phys. 98, 760 (2004).

${ }^{8}$ T. Y. Kim, N. M. Park, K. H. Kim, G. Y. Sung, Y. W. Ok, T. Y. Seong, and C. J. Choi, Appl. Phys. Lett. 85, 5355 (2004).
${ }^{9}$ L. W. Yin, Y. Bando, Y. C. Zhu, and Y. B. Li, Appl. Phys. Lett. 83, 3584 (2003).

${ }^{10}$ P. Giovanneschi and D. Dufresne, J. Appl. Phys. 58, 651 (1985).

${ }^{11}$ A. Rosencwaig, Annu. Rev. Biophys. Bioeng. 9, 31 (1980).

${ }^{12}$ G. C. Ratcliff, D. A. Erie, and R. Superfine, Appl. Phys. Lett. 72, 1911 (1998). 